\title{
La tecnología es protagonista: aplicaciones y servicios de la Biblioteca Rector Gabriel Ferraté
}

\author{
Por Javier Clavero, Miquel Codina y Andrés Pérez
}

\begin{abstract}
Resumen: Se presenta la experiencia tecnológica y los resultados de los últimos 5 años en la Biblioteca Rector Gabriel Ferraté de la Universitat Politècnica de Catalunya. Se hace una descripción de los recursos humanos destinados a la realización de los proyectos y del método de trabajo que se emplea. Los proyectos descritos abarcan campos tan variados como los servicios móviles, la gestión interna de colecciones, la gestión de espacios, la web 2.0, etc.

Palabras clave: Tecnología, Web, Aplicaciones móviles, Biblioteca universitaria, Videojuegos en bibliotecas, Universitat
\end{abstract} Politècnica de Catalunya.

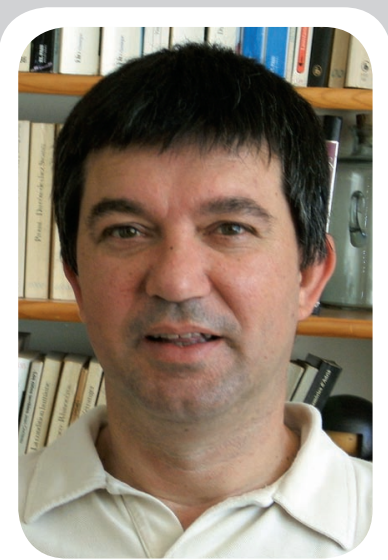

Miquel Codina, diplomado en biblioteconomía y documentación y licenciado en geografía e historia por la Univ. de Barcelona, es director de la Biblioteca Rector Gabriel Ferraté de la Univ. Politècnica de Catalunya (UPC).

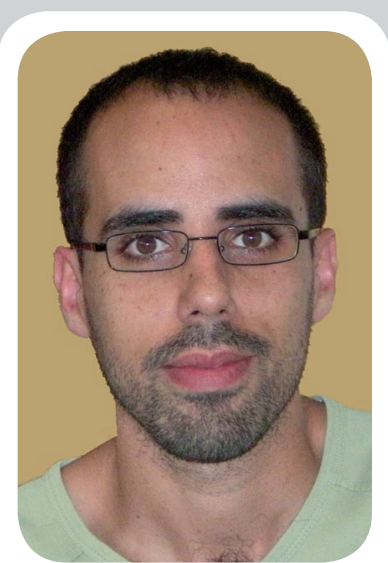

Andrés Pérez, diplomado en biblioteconomía y documentación por la Univ. de Barcelona y licenciado en documentación por la Univ. Oberta de Catalunya, es responsable de la Unidad de Servicios Digitales de la BRGF de la UPC.

Title: Technology as a protagonist: applications and services at the Rector Gabriel Ferraté IIbrary

Abstract: The Rector Gabriel Ferraté Library of the Universitat Politècnica de Catalunya presents its technological experience and outcomes of the last 5 years. The article also describes the human resources used for the implementation of the projects and the working method usually followed. The included projects cover various fields such as mobile services, collection management, building management, web 2.0, etc.

Keywords: Technology, Web, Mobile applications, University library, Gaming in libraries, Universitat Politècnica de Catalunya.

Clavero, Javier; Codina, Miguel; Pérez, Andrés. “La tecnología es la protagonista: aplicaciones y servicios de la Biblioteca Rector Gabriel Ferratê". El profesional de la información, 2010, enero-febrero, v. 19, n. 1, pp. 63-69.

DOI: 10.3145/epi.2009.nov.09

\section{Contexto}

\section{LA BIBLIOTECA RECTOR} GABRIEL FERRATÉ (BRGF) está ubicada en el Campus Nord de la Universitat Politècnica de Catalunya (UPC).

http://bibliotecnica.upc.edu/bib160

Presta sus servicios a unos 8.000 usuarios pertenecientes a la Facultat d'Informàtica, la Escola Tècnica Superior d'Enginyeria de Telecomunicació y a la Escola Tècnica Superior d'Enginyeria de
Camins, Canals $i$ Ports, a $22 \mathrm{de}-$ partamentos de la UPC y al colectivo del personal de administración y servicios de dichas unidades así como a una buena parte de los servicios generales de la Universidad (servicio de personal, servicio de economía, etc.).

Es evidente que, por las materias objeto de estudio en el campus, nos encontramos ante un escenario altamente tecnológico donde los usuarios acostumbran a dominar las herramientas TIC y donde los últi- mos avances y novedades del sector rápidamente encuentran mercado y público consumidor.

En este entorno la $B R G F$ apuesta por una alerta tecnológica continua que le permita conocer las tendencias, evaluarlas y aplicarlas en los casos en que aporten mejoras.

\section{Opción}

En 2004 la dirección de la $B R G F$ atendiendo a la nueva situación que había creado la difusión de internet y en previsión de que el 
avance tecnológico de servicios y aplicaciones fuera un elemento crítico de éxito, consiguió incorporar personal informático estable y cualificado a su plantilla. De esta manera se configuró la estructura actual de la Unidad de Servicios Digitales (USD): un bibliotecario con conocimientos TIC como responsable, un ingeniero informático superior como desarrollador de aplicaciones y administrador de sistemas, un técnico especialista que realiza tareas de apoyo y mantenimiento y 3 becarios con conocimientos informáticos y multimedia asignados a los diferentes proyectos en marcha.

A partir de este momento se produjo un salto cualitativo (por la calidad de los servicios que se empezaron a prestar) y cuantitativo (por la cantidad de servicios que se han podido proporcionar).

En 2008 se reforzó esta dinámica con la creación de un grupo de trabajo, el BiT (Biblioteca y Tecnología), que realiza un seguimiento constante de algunos campos tecnológicos que creemos de especial relevancia y aplicación en nuestra biblioteca como son: los avances en hardware, los gadgets (cualquier tipo de dispositivo electrónico novedoso), el software libre y la web 2.0. El grupo cuenta con la participación de profesionales del resto de las unidades de la Biblioteca ya que se pretende dar un enfoque transversal y amplio a los temas que se abordan.

\section{Método}

Enfocamos el trabajo en base a una planificación de objetivos anual que deja siempre un margen razonable para abordar los imprevistos que surgen.

Dividimos los proyectos en 2 categorías:

- Externos: los que se hacen públicos y que redundan en un nuevo servicio (o una mejora de uno ya existente) para los usuarios de la biblioteca.
- Internos: aquellos que llevamos a cabo para mejorar las condiciones de trabajo del personal de la biblioteca. Es importante tener en cuenta que una de nuestras prioridades es que el usuario interno dedique todo su tiempo a su trabajo específico y que la tecnología que se utilice no sea un freno para la consecución de sus fines.

Para la realización de ambos tipos de proyectos siempre contamos con la colaboración y supervisión de profesionales especializados en los diferentes campos (investigación, docencia, gestión administrativa, etc.) de manera que intentamos romper la cadena encargo-desarrollo-explotación y sustituirla por encargo-planificación conjunta-desarrollo-testeo-rediseño-explotaciónevaluación.

\section{Resultados}

A continuación realizamos un breve repaso de nuestra actividad actual:

\section{Sitio web}

En estos últimos años hemos conseguido sustituir un sistema manual de mantenimiento basado en plantillas por un sistema mixto que permite distribuir la generación de contenidos entre las personas responsables de los mismos para aquellas páginas que experimentan cambios frecuentes, así como para la página principal. Hemos incorporado elementos propios de la web 2.0 como las encuestas y un colaborativo, el OoohWeb!, para la recolección de enlaces de interés 2.0, mediante el cual los usuarios nos proponen sus recursos de interés.

http://bibliotecnica.upc.es/bib160 http://bibliotecnica.upc.es/bib160/ serveis/ooohweb/

\section{Web móvil}

La última implementación de importancia, antes de la migración inminente a Drupal prevista para el primer trimestre de 2010, ha sido el remake de los contenidos y su adaptación para la consulta en móviles de gama alta y pdas, de la web de la Biblioteca. Cabe destacar de este proyecto la adaptación paralela del catálogo de las bibliotecas de la UPC (basado en Millennium) para la consulta en estos dispositivos.

\section{http://bibliotecnica.upc.es/bib160} http://flas.upc.edu/brgf

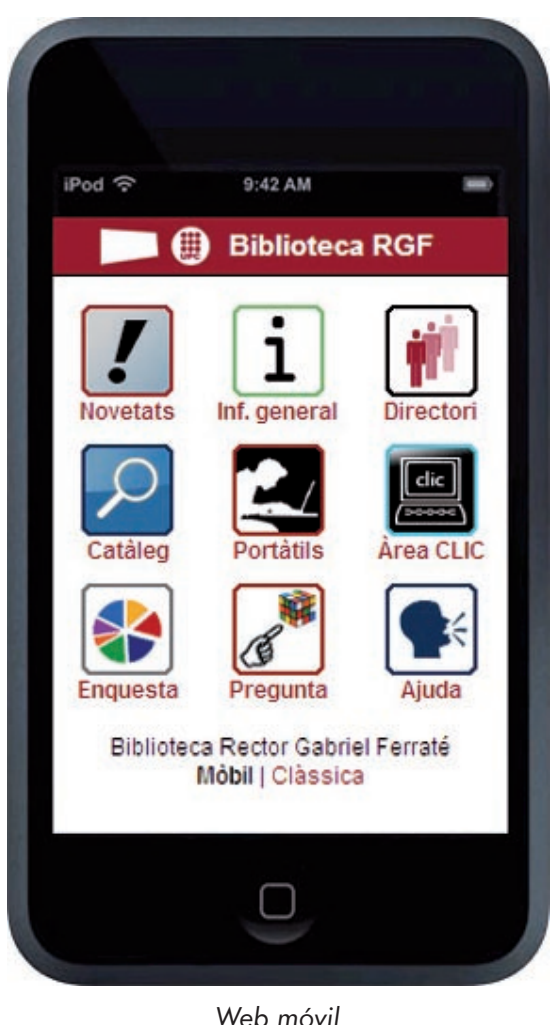

\section{Herramientas de búsqueda}

Para facilitar la búsqueda de información en los diferentes depósitos, portales y catálogos de las bibliotecas de la $U P C$, la $B R G F$ dispone de dos aplicaciones: la barra de herramientas $L i b X$ y un widget para Netvibes e iGoogle. LibX es gratuita para los principales navegadores, Firefox e Internet Explorer y especialmente diseñada para bibliotecas. Esta barra proporciona al usuario que se la instala, accesos directos a las principales webs que se deseen, además de un cuadro de texto que hace posible lanzar búsquedas contra las principales fuentes de información (catálogos, depósitos, etc.) que se hayan con- 
figurado. También permite realizar búsquedas en el propio catálogo de la institución desde sitios web como Amazon, Barnes \& Noble y Google, mediante un icono que la propia barra inserta en la web en cuestión. De esta forma, cuando se busca un libro en Amazon -u otros- se puede, mediante el icono que se encuentra al lado del título, saber si está disponible en alguna de las bibliotecas de la UPC. Por otro lado tenemos el widget creado para Netvibes e $i$ Google que, de forma parecida a la barra $\operatorname{LibX}$, permite a los usuarios realizar búsquedas sobre las principales fuentes de información de las bibliotecas de la UPC directamente desde sus escritorios virtuales y sin necesidad de instalar nada.

http://bibliotecnica.upc.es/bib160/ serveis/eines/

http://libx.org

\section{U-WIN}

Es un portal que amplía la oferta de servicios incluyendo el $g a$ ming. A la vez se intenta mostrar la producción de videojuegos de la $U P C$ y apostar por estos conteni- dos como materiales pedagógicos, abriendo la puerta a un modelo de biblioteca más social donde el esparcimiento también tiene cabida. U-WIN además cuenta con un espacio físico donde jugar, ubicado en la planta 0 de la Biblioteca.

\section{http://bibliotecnica.upc.edu/uwin}

\section{Avisos vía sms}

Es una herramienta de gestión interna. Consciente de la importancia del móvil en el día a día, la $B i$ blioteca apuesta por complementar algunos servicios con el envío de sms relativos a distintos servicios a los usuarios que lo soliciten. Esto se realiza mediante una aplicación web denominada $g S M S$ creada por UPCNet, empresa encargada de los servicios informáticos de la UPC.

\section{Área Clic}

La Biblioteca dispone de un aula con 24 ordenadores que pone a disposición de sus usuarios. Para poder hacer uso de estos equipos los usuarios tienen que registrarse en el mostrador de préstamo donde obtienen su nombre de usuario y contraseña; posteriormente pueden cambiar esta

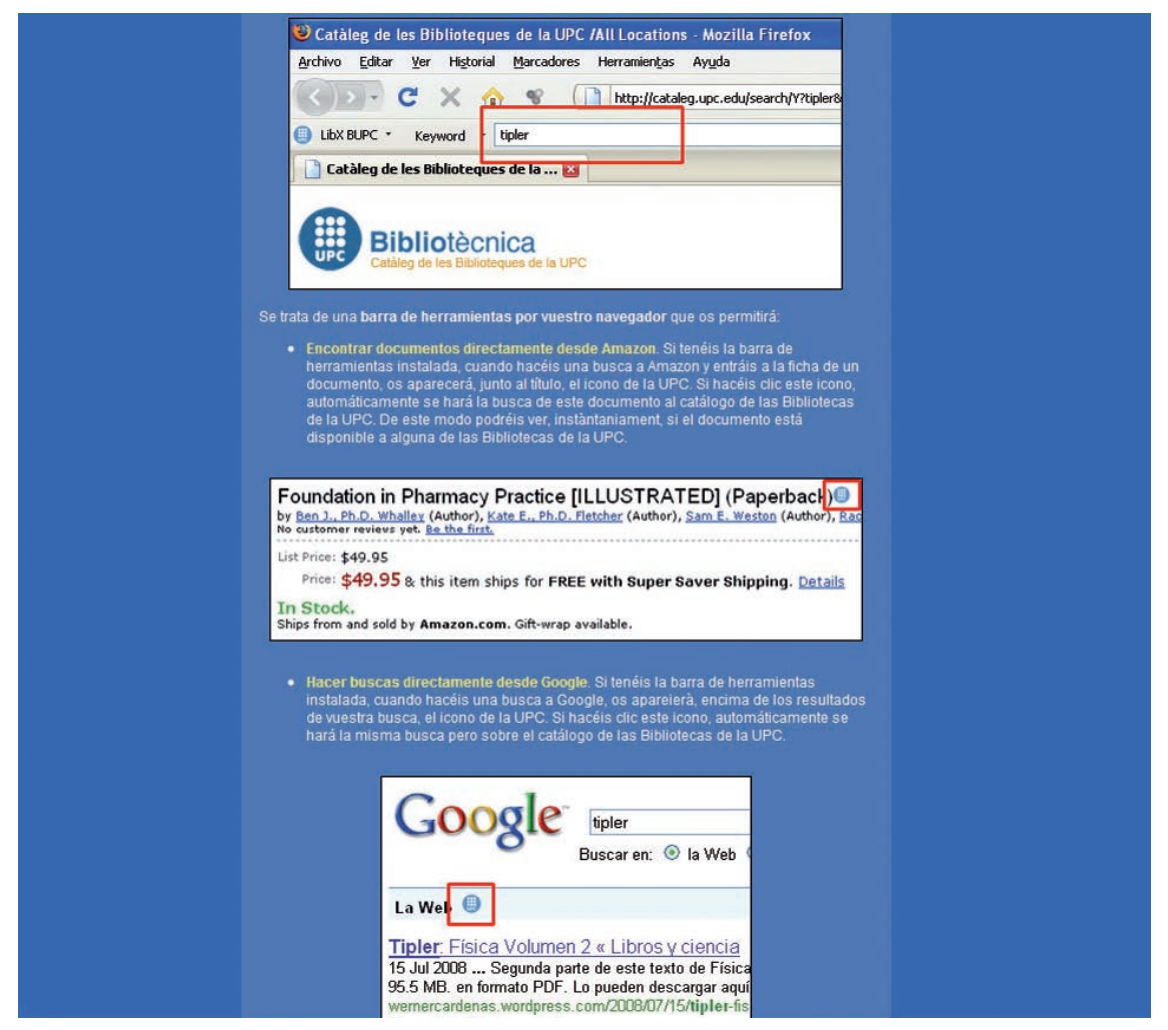

Herramientas de búsqueda (traducción automática a través de InterNostrum) contraseña desde la web de la Biblioteca. El software instalado en estos ordenadores es una recopilación del usado en las diferentes aulas informáticas de las 3 escuelas a las que se da servicio y anualmente se programa una revisión del mismo para realizar las actualizaciones pertinentes e instalar los nuevos programas que puedan ser necesarios. La gestión, tanto del software como de la validación de los usuarios, se realiza mediante el sistema Rembo, instalado en el servidor de la Biblioteca. El propósito es tener un aula útil para los usuarios pero que no requiera un mantenimiento permanente por parte del personal de la BRGF. Contar con este sistema ha supuesto un gran salto en la calidad del servicio ya que nos garantiza la disponibilidad de los equipos así como una gran flexibilidad para introducir cambios.

http://bibliotecnica.upc.es/bib160/ serveis/area_clic/

\section{Códigos QR (Quick Response)}

Se trata de una codificación parecida a un código de barras pero que permite almacenar muchísima más información, tanto textual como numérica. Es un sistema innovador que hace posible el almacenamiento de gran cantidad de datos en una imagen relativamente pequeña. Los códigos $Q R$ están íntimamente relacionados con los móviles ya que para extraer la información que contienen se necesita un móvil con cámara y una pequeña aplicación que se puede descargar de forma gratuita de muchas webs. Concretamente se usan códigos $Q R$ en algunos carteles de la Biblioteca y en la ficha con los datos de acceso al Área Clic que se entrega a los usuarios cuando se registran en el servicio. La idea es que, en ambos casos, el usuario pueda hacer uso del $Q R$ para almacenar la información en su móvil sin necesidad de apuntarla en un papel.

http://bibliotecnica.upc.es/bib160/ serveis/qr/ 


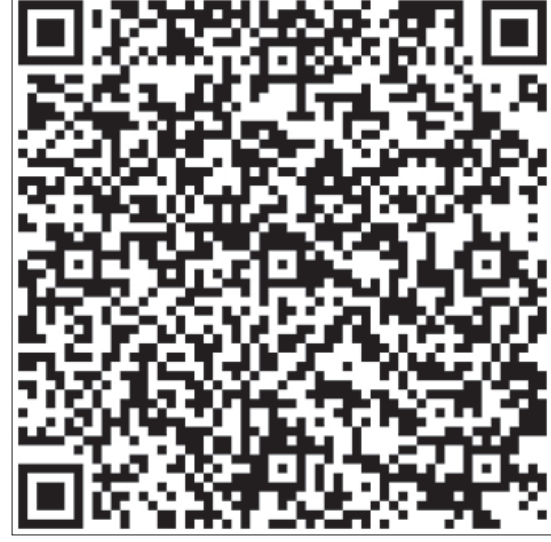

Códigos QR

\section{Préstamo de lectores de libros} electrónicos

En su iniciativa de potenciar las nuevas tecnologías en el entorno bibliotecario y de la educación superior, la $B R G F$ puso en funcionamiento en noviembre de 2008 un servicio de préstamo de lectores de libros electrónicos. El servicio nació de un acuerdo entre la Biblioteca y la Facultad de Informática para adquirir este tipo de equipos para la lectura de proyectos de final de master por parte de los profesores del tribunal evaluador. Se dispone de 5 lectores de libros electrónicos iRex iLiad 2nd edition que se prestan desde el catálogo por un período de 10 días. Junto con el dispositivo se presta el lápiz táctil (los equipos disponen de pantalla táctil que permite realizar anotaciones) y una tarjeta de memoria con contenidos variados para su lectura. Estos contenidos corresponden a libros técnicos de Edicions UPC, la editorial de la Universidad, libros específicos de informática de la editorial $O$ 'Reilly y un conjunto de novelas. Dada la buena acogida del servicio se está trabajando tanto en ampliar los contenidos disponibles como en la adquisición de más equipos. Además, el Servicio de Bibliotecas y Documentación, está valorando la posibilidad de extender este servicio a otras bibliotecas de la $U P C$.

http://bibliotecnica.upc.es/bib160/ serveis/ebooks/

\section{Préstamo de portátiles}

Se dispone de 23 portátiles que los usuarios pueden obtener en préstamo durante un período de tiempo máximo de 3 horas, ampliables en el caso de que no haya reservas pendientes. No se trata de un servicio exclusivo de la $B R G F$, sino que se ofrece en todas las bibliotecas de la $U P C$ ya que fue una iniciativa del Servicio de Bibliotecas y Documentación. Para utilizar el servicio los usuarios deben dirigirse al mostrador de préstamo, identificarse como miembro de la Universidad y, una vez aceptada la normativa, se les entrega uno de los portátiles. El uso de estos equipos está restringido al interior del edificio, pero se está valorando prestarlos para su uso en cualquier lugar. Tanto el sistema de préstamo como el de reservas que pueden realizar los usuarios se gestionan mediante una aplicación de creación propia. Esto es así debido a que las limitaciones del sistema de catálogo del que se disponía en el momento de la creación del servicio no permitían realizar los préstamos con las condiciones que se habían determinado. Se trata de uno de los servicios con más éxito de todas las bibliotecas, en especial de la $B R G F$, y se está trabajando para ampliar el número de equipos.

http://bibliotecnica.upc.es/bib160/ serveis/prestec_portatils/

\section{Reserva de salas para trabajo en grupo}

Una de las principales peticiones que se habían recibido siempre en la $B R G F$ por parte de los usuarios era la creación de aulas para trabajo en grupo. En 2008 la dirección obtuvo los fondos necesarios para construir 6 salas de trabajo en grupo, que se ubicaron en la planta 2. Con el fin de potenciar aún más su uso y el trabajo colaborativo que se plantea en el EEES, en dos de estas salas se instalaron equipos multimedia formados por una pantalla de alta definición y un ordenador.
Durante el primer año en funcionamiento las salas se han convertido en uno de los servicios estrella, con más de 6.500 reservas. Si un usuario desea una sala, previamente ha de realizar una reserva desde la web de la Biblioteca para, posteriormente, dirigirse al mostrador de la planta 2 donde, mostrando su carné universitario, el bibliotecario o técnico de la planta le proporcionará la llave de la sala reservada. Además del módulo de reservas que utilizan los usuarios la aplicación que gestiona las salas dispone de un módulo de administración, únicamente accesible para el personal de la Bibliote$c a$, que permite controlar, en cada momento, quien dispone de cada sala, las reservas pendientes y los usuarios sancionados por mal uso. Dado el gran éxito del servicio, se construyeron 7 salas más en la planta 1 en 2009.

http://bibliotecnica.upc.es/bib160/ serveis/sales

\section{CanalBIB y aplicaciones integra- das}

Es una herramienta de gestión interna. El CanalBIB es un servicio implementado por el Servicio de Bibliotecas y Documentación y la empresa PuntXarxa para dar más visibilidad a las informaciones y novedades relacionadas con la $U P C$ y en concreto con las bibliotecas. El servicio dispone de pantallas de alta resolución y de gran tamaño (de 42" a 52") repartidas por las bibliotecas de la $U P C$, en las que se proyecta, desde un ordenador conectado a la red, un carrusel de noticias relacionadas con la Biblioteca donde se encuentra la pantalla y con la escuela o campus. Estas noticias son gestionadas por el personal de la propia biblioteca o por el del $S B D$ de la $U P C$. Un inconveniente de este sistema es que las informaciones deben darse de alta manualmente desde la interfaz de administración para que se visualicen en la pantalla. Es por ello que la Unidad de Servicios Digitales 


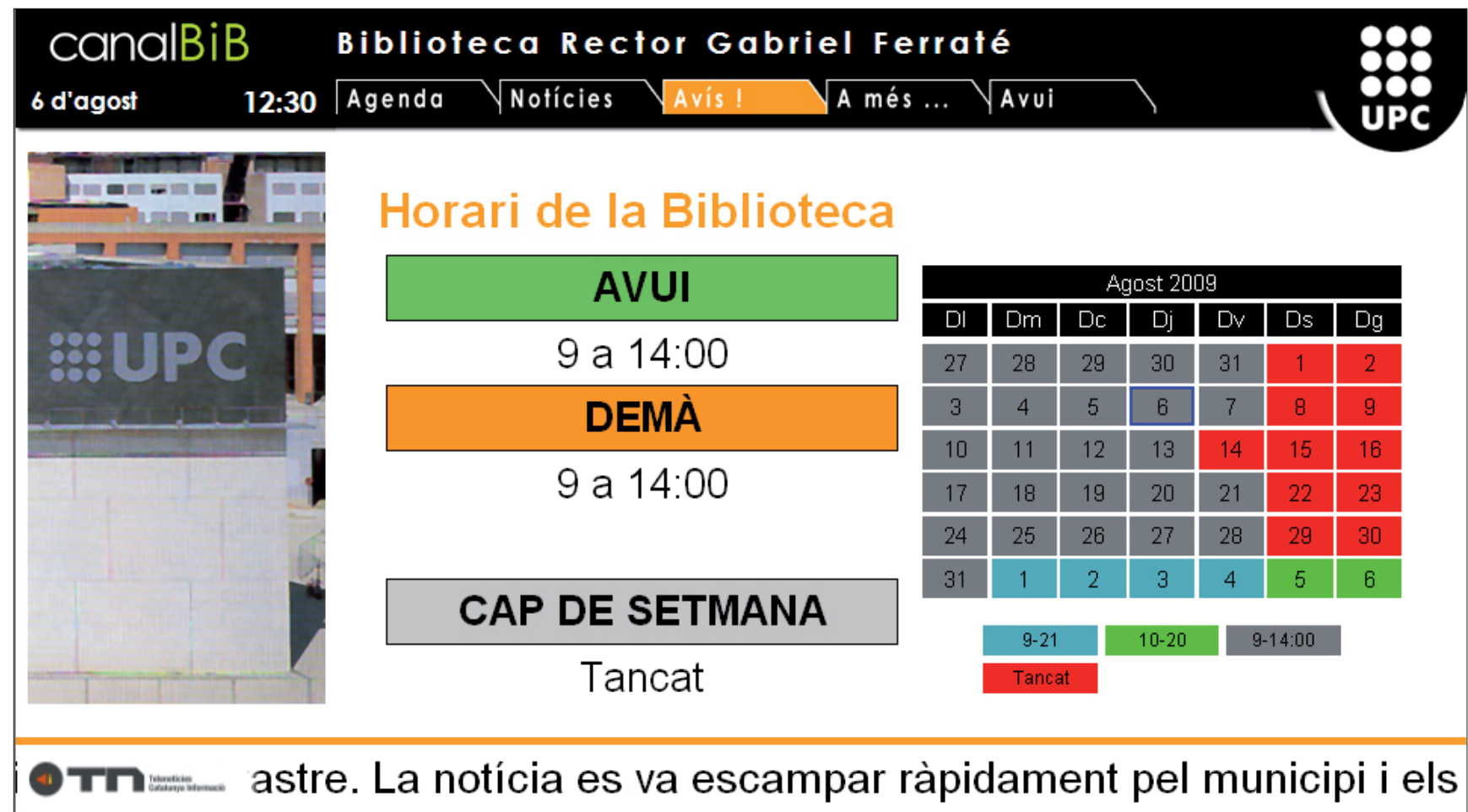

CanalBIB

(USD) de la BRGF implementa una serie de aplicaciones integradas en el CanalBIB que permiten de forma automática y sin necesidad de intervención humana mostrar informaciones relevantes de la Biblioteca como horarios, disponibilidad de portátiles, disponibilidad de salas, etc. Actualmente estos aplicativos son usados por todas las bibliotecas de la UPC a través de sus respectivas pantallas de CanalBIB.

\section{Laboratorio Virtual de Idiomas (LVI)}

Es una apuesta del $S B D$ de la $U P C$ por la difusión y autoaprendizaje de los idiomas. El LVI es un sitio web donde se ofrece gran cantidad de recursos, gratuitos y de pago, para el autoaprendizaje de idiomas, a los que puede acceder cualquier usuario desde cualquier parte del mundo. La selección de los recursos se realiza cuidadosamente para intentar ofrecer la máxima calidad. Los recursos de pago se ponen a disposición de los usuarios mediante la plataforma Citrix que hace posible el acceso a aplicaciones instaladas en un servidor como si estuvieran instaladas en su propio ordenador. Cabe destacar que este es un proyecto creado al $50 \%$ entre la $B R G F$, aportando el personal informático, y el $S B D$ aportando el personal bibliotecario y los medios económicos necesarios.

http://bibliotecnica.upc.edu/lvi/

\section{Punto de información digital}

Aplicación de uso local en estaciones de trabajo de la BRGF. Hasta hace pocos meses la Biblioteca ofrecía en cada una de sus plantas una serie de folletos informativos de sus diferentes servicios. Estos folletos no eran muy apreciados por

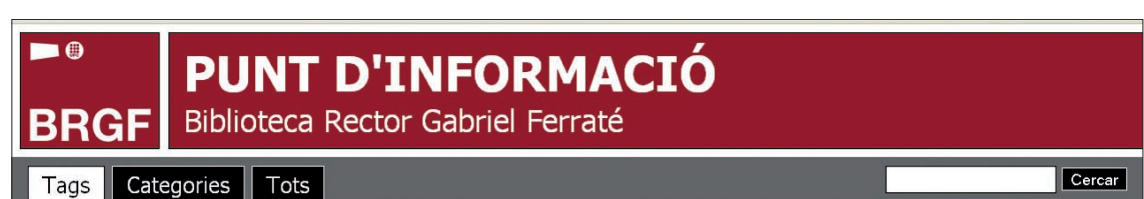

\begin{tabular}{l|l|l|} 
Tags & Categories Tots \\
\hline
\end{tabular}

còpia bibliografia dates localitzar préstec jazz cercar format horari enginyeria civil informàtica benvinguda articles consultar autor sumaris subscripció novel'les renovació títol caì)

biblioteca documents revistes àrea CLIC colleccions referències material docent electrònica telecomunicacions camins col-lecció recursos serveis normativa reserva gènere musica ciència-ficció informació bibliogràfica 
los usuarios y requerían un mantenimiento elevado, tanto de papel como de tiempo de reposición. Para acabar con este problema y potenciar el uso de estas ayudas se creó el Punto de Información Digital. Se trata de terminales donde el usuario, mediante una interfaz creada específicamente para ello, puede buscar y consultar estas informaciones básicas y ayudas de forma económica y fácil. Se puede acceder mediante una nube de tags, por materias o por un buscador a las diferentes ayudas que se ofrecen. Es posible también enviar dichas ayudas (ficheros pdf) por correo electrónico a la dirección que se especifique.

\section{Noticiario de la $B R G F$}

Mensualmente la Biblioteca envía a los profesores y doctorandos de los centros del Campus Nord un boletín informativo con novedades que se consideran de interés para los miembros de estos colectivos. La principal diferencia respecto a otros boletines de otras bibliotecas es que no sólo se adjuntan novedades sobre la $B i$ blioteca y su entorno sino también novedades temáticas o especializadas sobre aspectos relacionados con alguna de las especialidades del Campus. Se extraen de prensa especializada o portales temáticos de internet. Cualquier miembro del personal puede sugerir una noticia: para ello se dispone de una aplicación que permite hacer llegar esta novedad de forma automática al responsable de la edición del boletín. Por otro lado, el boletín se envía a los suscriptores mediante un correo electrónico que contiene la información en diversos formatos para que pueda ser leída de manera atractiva en el mayor número de clientes de correo. El boletín mensual y los números anteriores también se pueden consultar desde la web de la Biblioteca y dispone de suscripción RSS.

http://bibliotecnica.upc.es/bib160/ serveis/noticiari_brgfl

\section{Netvibes}

Es una herramienta de gestión interna. Como es sabido, Netvibes es una aplicación gratuita de escritorio virtual que permite acceder desde una única pantalla a información de otras aplicaciones y portales mediante unos elementos denominados widgets. El principal propósito de un escritorio virtual es el acceso a una gran cantidad de información de un solo vistazo y de una forma fácil y cómoda, evitando tener que visitar múltiples portales y aplicaciones. En los mostradores de las diferentes plantas de la $B R G F$ son habituales las consultas sobre informaciones generales como horarios, portátiles disponibles, direcciones del campus, etc. Hasta el momento el personal tenía que acudir a diferentes webs para satisfacer estas demandas y es por esto que se pensó que se podrían concentrar todas estas informaciones en un escritorio virtual. La Biblioteca abrió una cuenta en Netvibes y estructuró con widgets propios, creados por la USD y terceros, un escritorio virtual que concentrara estas informaciones y permitiera al personal de los mostradores satisfacer las consultas de los usuarios de un solo vistazo. Este escritorio virtual esta configurado como la página de inicio de todos los ordenadores de los mostradores.

\section{http://www.netvibes.com}

\section{BibAdmin}

Es una herramienta de gestión interna. Se trata de una aplicación web que permite gestionar de forma integral y unificada todos los portales, aplicaciones y servicios. No sólo se trata de un sistema de gestión de contenidos sino que también proporciona todo un entorno que hace posible configurar parámetros y opciones (impresoras, carpetas compartidas, fondos de pantalla, etc.) de los ordenadores de uso público y también de los ordenadores de uso interno, e incluso la creación automática bajo demanda de la cartelería de la $B i$ blioteca. El propósito principal de esta aplicación de creación propia, es que el personal tenga acceso y control desde una única interfaz a todo aquello que necesita en su trabajo habitual, evitando que tenga que acudir a diversas aplicaciones y pantallas.

\section{Base de datos temática}

Es una herramienta de gestión interna. La catalogación es una de las tareas básicas, y la mejora de las herramientas de trabajo de los bibliotecarios dedicados a esta tarea una de nuestras prioridades. La base de datos temática es una aplicación que se creó para recopilar las diferentes materias usadas por los catalogadores de la $B R G F$ en los sistemas de la UPC y las relaciones que hay entre ellas, tanto jerárquicas como de equivalencia, además de relacionar las diversas materias con los topográficos a los que se corresponden, la ubicación de éstos en la Biblioteca y una serie de palabras clave identificativas de cada materia. El propósito principal de esta aplicación era ayudar a la catalogación de los nuevos materiales, por un lado normalizando la nomenclatura de las materias $\mathrm{y}$, por otro, homogeneizando el uso de las mismas en casos similares. Además de esto también se pensó en crear productos relacionados, que tendrían como núcleo esta base de datos, como: la generación automática de los carteles donde el usuario puede consultar la ubicación de un topográfico dentro del edificio o bien una aplicación que permitiera al usuario saber la ubicación de un libro directamente sin necesidad de un cartel intermediario. Esta base de datos es una aplicación muy utilizada y apreciada por el personal catalogador y cabe decir que su construcción y concepción no fue fácil ya que la cantidad de materias de que dispone la $B R G F$ es muy elevada. 


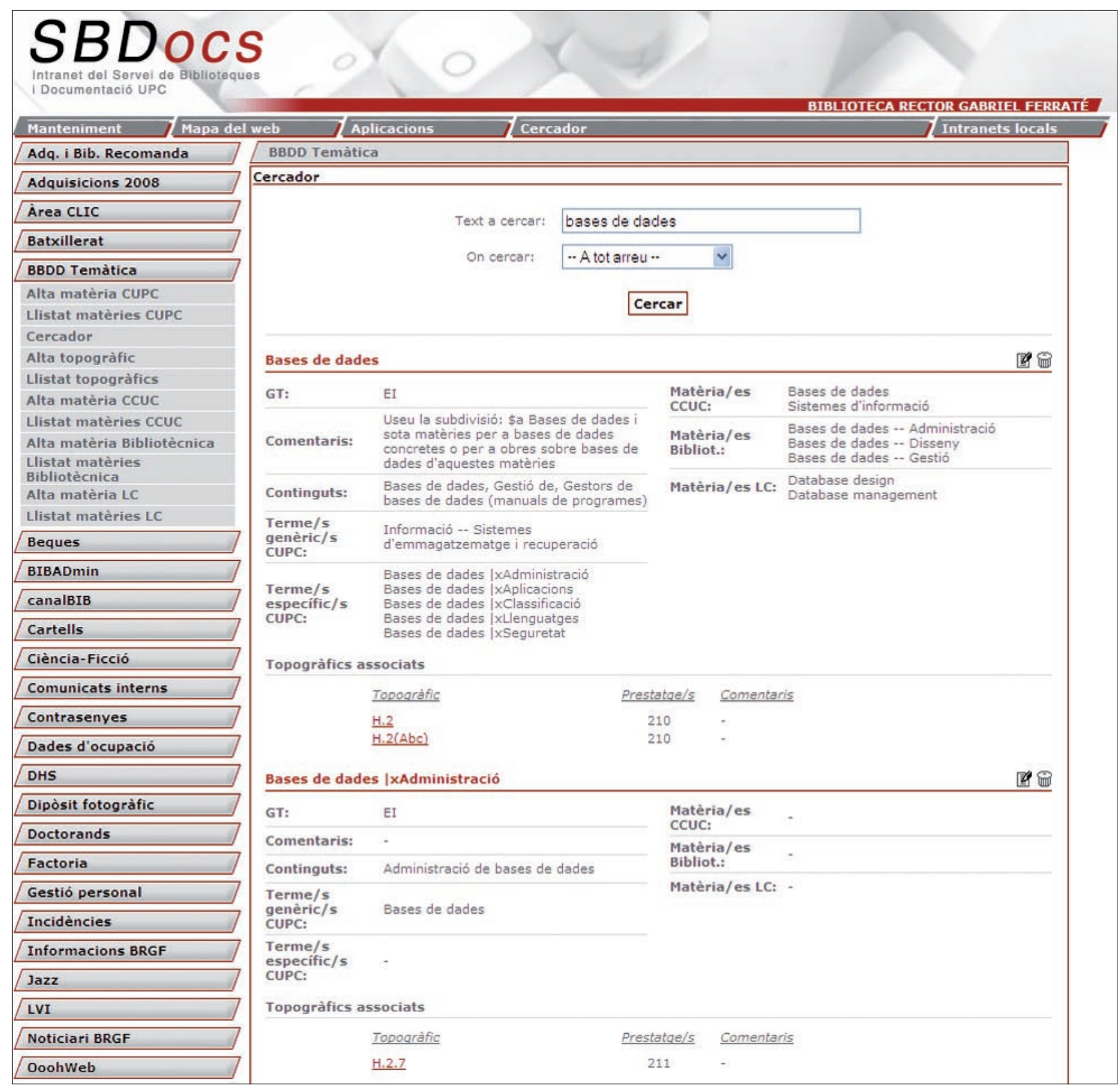

Base de datos temática

\section{Punto y seguido}

Las aplicaciones descritas se encuentran en evolución debido a la implementación del nuevo sistema de gestión de contenidos (basado en Drupal) que nos está empujando a replantear diversas aplicaciones y que también nos abre puertas para la realización de algunas nuevas. En los próximos meses se concretará la migración y se podrán ver nuevas herramientas.

Para ampliar la información ofrecida se recomienda consultar la web de la $B R G F$ o bien contactar directamente con los autores.

\section{Bibliografía de referencia}

Arroyo-Vázquez, Natalia. "Web móvil y bibliotecas". El profesional de la información, 2009, marzo-abril, v. 18, n. 2, pp. 129-136.

Clavero, Javier; Codina, Miquel; Pérez, Andrés; Serrat-Brustenga, Marta. "Estudio de caso de préstamo de libros electrónicos". El profesional de la información, 2009, marzo-abril, v. 18 , n. 2, pp. 237-241.

Freire, Juan. "Redes sociales: ¿modelos organizativos o servicios digitales?". El profesional de la información, 2008, noviembre-diciembre, v. 17 , n. 6 , pp. 585-588.

Gil, Adriana. Els videojocs. Barcelona: UOC, 2008. 140 pp.

Hassan, Yusef; Martín-Fernández, FranciscoJ.; Iazza, Ghzala. "Diseño web centrado en el usuario: usabilidad y arquitectura de la información”. Hipertext.net, 2004, mayo. http://www.hipertext.net/web/pag206.htm

Johnson, Steven. Everything bad is good for you: how popular culture is making us smarter. London: Penguin, 2005.

McKiernan, Gerry. Mobile libraries. http://mobile-libraries.blogspot.com/

Javier Clavero, Miquel Codina, Andrés Pérez. Biblioteca Rector Gabriel Ferraté (BRGF), Universidad Politécnica de Catalunya (UPC). Jordi Girona, 1-3. 08034 Barcelona.

javier.clavero@upc.edu miquel.codina@upc.edu andres.perez@upc.edu 